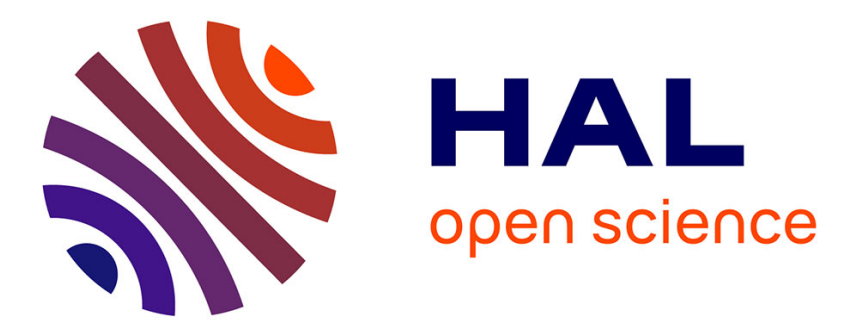

\title{
Quel leader et business model ouvert pour les écosystèmes-plateformes de type NFC ?
}

Amel Attour

\section{To cite this version:}

Amel Attour. Quel leader et business model ouvert pour les écosystèmes-plateformes de type NFC ?. Revue management \& avenir, 2014, 73, pp.33-53. 10.3917/mav.073.0033 . halshs-01104079

\section{HAL Id: halshs-01104079 \\ https://shs.hal.science/halshs-01104079}

Submitted on 16 Jan 2015

HAL is a multi-disciplinary open access archive for the deposit and dissemination of scientific research documents, whether they are published or not. The documents may come from teaching and research institutions in France or abroad, or from public or private research centers.
L'archive ouverte pluridisciplinaire $\mathbf{H A L}$, est destinée au dépôt et à la diffusion de documents scientifiques de niveau recherche, publiés ou non, émanant des établissements d'enseignement et de recherche français ou étrangers, des laboratoires publics ou privés. 


\title{
Quel leader et business model ouvert pour les écosystèmes-plateformes de type NFC ${ }^{1}$
}

\author{
Amel ATTOUR \\ GREDEG (UMR 7321), Université de Nice Sophia-Antipolis \\ Amel.attour@gredeg.cnrs.fr
}

\begin{abstract}
Résumé. Cet article s'intéresse aux écosystèmes de type 'plateformes' (Koening, 2012). Il focalise en particulier sur le cas d'écosystèmes naissants où le leader et la plateforme pivot ne sont pas préalablement identifiés. Il applique pour cela les méthodes de prototypage stratégique (Chanal et al. 2011) au cas d'un écosystème du Near Field Communication naissant dont il caractérise le business model. A l'appui d'un cadre théorique mobilisant la littérature sur les stratégies de plateforme et les business model ouverts, les analyses de la proposition de valeur et de l'architecture de valeur du cas NFCampus permettent de montrer premièrement que l'émergence des écosystèmes du NFC relève de l'articulation de deux types de plateformes pivots telles qu'elles sont définies par Boudreau et Lakhani (2009). Comme le montre deuxièmement cet article, le positionnement dans le rôle leader d'un acteur pivot est identifié à travers le business model ouvert (BMO) et le rôle de sa plateforme. L'acteur identifié leader à la naissance de l'écosystème peut en revanche être détrôné en phases d'expansion et d'autorité (après diffusion de l'innovation auprès des utilisateurs finaux).
\end{abstract}

Mots clefs: business model ouvert, écosystèmes numériques, stratégies de plateforme, NFC.

\section{Which leader and which open business model for NFC platform-based ecosystems?}

\begin{abstract}
The paper focuses on platform ecosystems (Koening, 2012). It focuses in particular on the case of emergent platform-ecosystems where both the leader and the platform are not beforehand identified. In this objective, we apply the business model innovation's methods (Chanal et al. 2011) to the case of Near Field Communication ecosystem. The elaborated business model is then analyzed thanks to our theoretical framework mobilizing the literatures on platform strategies and on open business model. Our results underline first that the emergence of a NFC ecosystem is possible when two open platforms articulate the creation and share of the ecosystem's value mechanisms. The open business model of the hub platform gives to its proprietary the leadership. However, this leadership has not the same role in the birth phase of the ecosystem and in the expansion and authority phases.
\end{abstract}

Keywords: open business model, digital ecosystems, platform strategies, NFC.

\footnotetext{
${ }^{1}$ Cet article a fait l'objet de deux communications. Une communication aux journées de recherche du BETACNRS-UMR 7522 organisées en mai 2012 (document de travail BETA ${ }^{\circ}{ }^{2012}$-12) et une deuxième communication à l'école d'été organisée par le réseau de recherche sur l'innovation (RRI) organisée les 29 août - $1^{\mathrm{er}}$ septembre 2012. Le présent projet d'article est une version entièrement retravaillée et n'est pas une reproduction des deux communications ci-dessus citées.

*L'auteur souhaite remercier ici les deux évaluateurs anonymes de la revue pour leurs commentaires et recommandations avisées ainsi qu'un relecteur pour son regard critique. Les insuffisances subsistantes restent de la responsabilité de l'auteur.
} 


\section{Introduction}

Situé entre un réseau stratégique complexe et une communauté d'acteurs aux rôles complémentaires (Gueguen et Passebois-Ducros, 2011), un écosystème d'affaires est une forme d'organisation des échanges, une structure ou un cadre institutionnel à même de gérer les relations entre plusieurs acteurs engagés dans un processus d'innovation collectif plus ou moins ouvert. Selon la taille de l'organisation, le degré de contrôle (centralisé ou décentralisé) des ressources clés et le type d'interdépendances de ses acteurs hétérogènes, un écosystème d'affaires peut être agencé sous la forme de 'communauté de destin', de 'communauté de foisonnement', de 'système d'offre' ou de 'plateforme' (Koening, 2012, 2013). Similaire aux districts industriels, mais affranchie de la condition de l'ancrage territorial, une 'communauté de destin' prend la forme d'un système non centralisé dont les membres hétérogènes s'unissent involontairement (tels des naufragés) pour empêcher la modification des règles du jeu ou de l'équilibre du marché par un innovateur. Une communauté de destin est de fait caractérisée par la solidarité existentielle de ses acteurs membres. Plus nombreux et réunis autour d'une ressource collective qu'ils enrichissent, les membres des 'communautés de foisonnement' forment des pairs dont les contributions sont identifiables et isolables. Dans le cas des 'systèmes d'offre', une ou plusieurs ressources clés sont la propriété d'un acteur leader (mandant) qui délègue aux membres de l'écosystème (mandataires) la réalisation d'actifs préalablement définis dans leurs complémentarités. Cette logique de modularité caractérise également les écosystèmes de type 'plateforme' où un acteur leader ouvre, selon des règles précisées ex ante, l'accès d'un actif clé à des acteurs externes qui en enrichissent la valeur. Cet actif clé - la plateforme - peut prendre la forme d'un service ou d'un ensemble de solutions techniques favorisant la création d'activités de niche génératrices de valeur pour l'ensemble de l'écosystème (Moore, 1996 ; Iansiti et Levien, 2004 ; Isckia, 2009, 2011). Le développement de ces activités complémentaires relève ici d'initiatives indépendantes organisées (via la plateforme) selon un mode d'interdépendance de type pool.

Une attention particulière a été accordée par la littérature aux écosystèmes-plateformes. Les nombreux exemples, dont la plus grande majorité des plateformes étudiées relève du secteur des télécommunications, caractérisent la stratégie de leadership adoptée par une firme en situation d'échec de marché ou cherchant à asseoir une position dominante (Gawer et Cusumano, 2002 ; Iansiti et Levien, 2004 ; De Vogeleer et Lescop, 2011). Cette littérature met l'accent sur la relation étroite entre plateforme technologique et écosystème. En revanche, à l'exception de Parker et Alystyne (2005), Eisenmann et al. (2009), Evans et al. (2006), Gawer et Henderson (2007), Hagiu (2007a, 2007b, 2009), Isckia (2009, 2011), Isckia et Lescop (2009, 2013), De Vogeleer et Lescop (2011), Gawer et Cusumano (2012), très peu de travaux analysent les raisons faisant des plateformes technologiques la condition ou le point d'émergence d'un écosystème de type plateforme. Les rares travaux sur le sujet focalisent sur le cas d'écosystème existant et dont la plateforme fait ou a fait l'objet de construction par une seule firme destinée à endosser le rôle de leader.

Le présent article souhaite contribuer à cette littérature en considérant le cas des écosystèmes naissants. Il s'intéresse au cas des écosystèmes naissant tel que celui qui caractérise le nouveau secteur des télécommunications. L'accent est plus particulièrement porté sur les écosystèmes du Near Field Communication ${ }^{2}$ (NFC) où à la fois l'acteur leader et le service ou l'actif technique endossant le rôle de plateforme n'ont pas préalablement été identifiés. Au sein du nouvel écosystème des télécommunications les firmes sont segmentées en deux

\footnotetext{
${ }^{2}$ Le NFC est une technologie de «communication en champ proche», sans-fil, de courte portée et haute permettant l'échange d'informations entre deux périphériques distancés l'un de l'autre d'environ $10 \mathrm{~cm}$. La technologie NFC est une extension de la norme ISO/CEI 14443 standardisant les cartes de proximité utilisant la radio-identification (RFID), qui combinent l'interface d'une carte à puce et un lecteur au sein d'un seul périphérique (Wikipédia).
} 
groupes distincts (Basole et Rouse, 2008 ; Basole, 2009). D'un côté, les acteurs traditionnels du secteur des télécommunications, identifiés comme fondateurs technologiques du nouvel écosystème; de l'autre les nouveaux entrants. Trois fonctions peuvent être exercées par les acteurs de ces deux segments. Les firmes peuvent avoir un rôle de pivot (hub), de connecteur (bridge) ou d'intermédiaire (broker). Les firmes-connecteurs garantissent la mise en réseau et la connectivité de l'écosystème. Les firmes-intermédiaires mettent en relation deux segments de l'écosystème ou encore deux firmes ou groupes de firmes appartenant à un même segment (Iansiti et Levien, 2004 ; Iyer et al. 2006). Caractérisées par un nombre disproportionnel et important de liens avec les autres membres de l'écosystème, plusieurs firmes au sein du nouvel écosystème des télécommunications sont candidates au rôle de pivot. Plus précisément, cette fonction peut en effet être endossée par une ou plusieurs firmes, ou tout un segment de l'écosystème. Les travaux de Basole (2009) concluent par exemple sur le positionnement clef des fournisseurs de plateformes au sein du nouvel écosystème des télécommunications. Dans ces travaux une 'guerre de leadership' est pressentie au sein des fournisseurs de plateforme qui pourtant sont identifiés comme des acteurs connecteurs clef au sein de l'écosystème. La question de savoir «comment et quand» une firme se positionnera leader reste de fait entière et encore non résolue (Basole, 2009, p.13). Or la réponse à cette problématique est d'autant plus importante qu'elle permet de déterminer le business model d'un écosystème naissant.

Cet article propose de répondre à cette problématique à l'appui du cas « Nice Futur Campus » (NFCampus). Il pose la question de savoir comment identifier le ou les acteurs leader d'un écosystème-plateforme naissant. Comme le recommandent Chesbrough (2006) et Teece (2010), l'analyse du positionnement stratégique des acteurs au sein d'un écosystème doit d'abord être précédée par l'analyse des deux fonctions du business model de l'innovation : la proposition de valeur et l'architecture de valeur. Dans cette perspective, l'article applique dans le cadre d'une recherche ingénierique (Chanal et al. 1997) les méthodes de prototypage stratégique introduites par Chanal (2011) à un cas représentatif des écosystèmes du NFC : NFCampus (partie 2). Menée dans la partie 3 de cet article et analysée à l'aide d'un cadre théorique mobilisant la littérature sur les stratégies de plateformes et les business models ouverts (partie 1), la caractérisation des deux fonctions du business model de NFCampus montre que l'émergence des écosystèmes du NFC relève de l'articulation de deux types de plateformes partiellement ouvertes telles qu'elles sont définies par Boudreau et Lakhani (2009). La discussion de ces résultats permet de répondre à la question de Basole (2009) dans la partie conclusion de cet article.

\section{Les écosystèmes-plateformes : stratégie de leadership et business model ouverts}

Pour comprendre pourquoi les plateformes sont des intermédiaires importants au sein d'un écosystème, il convient de revenir sur leur rôle (1.1) et les business models qui leurs sont associés (1.3).

\subsection{Les plateformes technologiques, une stratégie de leadership}

Une plateforme technologique est la propriété d'une firme qui en ouvre l'accès à des acteurs externes en vue d'en enrichir la valeur. Quelle que soit sa forme (produits, services ou technologies), une plateforme technologique est amenée à évoluer avec son écosystème sous trois conditions (Gawer, 2009; Gawer et Cusumano, 2002, 2008, 2010,2012) : (i) il existe plusieurs firmes externes pouvant enrichir la valeur des composantes de la plateforme, (ii) la valeur pour le client est plus faiblement créée par l'acteur jouant le rôle d'assembleur des composantes de la plateforme que par les composantes elles-mêmes, (iii) les acteurs de 
composantes peuvent trouver des opportunités de marché différentes de celles de leur écosystème originel. Techniquement, son architecture est modulaire, i.e. composée de connecteurs et d'interfaces permettant à d'autres firmes d'implémenter à moindre coûts leurs produits, services ou technologies. Ces firmes sont dépendantes des ressources mises à disposition par le propriétaire de la plateforme, mais ne sont pas nécessairement liées par des relations clients-fournisseurs (Iyer et Davenport, 2008). Elles appartiennent à la même architecture de valeur et partagent une même stratégie de marché. La demande s'adresse en effet à l'ensemble des acteurs de l'écosystème qui considérés isolément auraient été confrontés à une demande inexistante ou à un échec de marché (Gawer et Henderson, 2007 ; De Vogeleer et Lescop, 2011). La valeur de la plateforme s'accroît en effet avec le nombre de produits ou services complémentaires et générateurs d'externalités de réseau ${ }^{3}$. Le produit, service ou usage final de la plateforme n'est par conséquent pas déterminé à l'avance et peut, parfois volontairement, être incomplet. Il dépend du niveau d'ouverture de la plateforme, en particulier de sa capacité à inciter une tierce partie à innover et à enrichir la proposition de valeur originelle.

En définitif, une plateforme est caractérisée par une forte adaptabilité et capacité à intégrer les innovations technologiques d'acteurs externes (Gawer et Cusumano, 2002). Elle fait pour cela l'objet d'innovation permanente en vue de maintenir sa position centrale au sein de l'écosystème d'une part, la nécessité pour les firmes externes de rester connectées à elle d'autre part (Moore, 1996 ; Iansiti et Levien, 2004 ; Gawer et Cusumano, 2012). Comme le précisent Iansiti et Levien (2004), la plateforme "should monitor the health of their ecosystem, promote reasonable business models and relationships, and invest in the kinds of platforms, technological components, and tools that enable third party productivity, diversity, and innovation. Furthermore, they should do so at terms that promotes the continued and sustainable growth of ecosystem participants », (p.58).

Dans cette perspective, plusieurs décisions stratégiques et technologiques sont étroitement liées à la plateforme qui incarne l'écosystème (Gawer et Cusumano, 2002, 2008 ; Evans et al. 2006 ; Iansiti et Levien, 2004a, 2006 ; Iyer et Davenport, 2008 ; Baldwin et Woodard, 2009 ; Isckia, 2011). Chaque phase du cycle de vie d'un écosystème est en effet caractérisée par un enjeu stratégique (Moore, 1996, p.103-109). Dans la phase pionnière (naissance de l'écosystème), un acteur visionnaire - leader - initie une trajectoire d'innovation collective et ouverte (Edouard et Gratacap, 2011; Isckia, 2011). Dans cette perspective, il met à disposition des membres de l'écosystème un système de valeurs, des règles, des objectifs économiques et une plateforme (Edouard et Gratacap, 2009). L'ouverture de la plateforme est source d'exploration de nouveaux espaces de marché pour les acteurs membres de l'écosystème (Isckia, 2011 ; De Vogeleer et Lescop, 2011). Le choix du degré d'ouverture de la plateforme relève par conséquent d'une décision stratégique cruciale. Comme le soulignent les travaux de Boudreau, Lakhani (2009) et Eisenmann et al. (2011), ce degré d'ouverture détermine le business model de la plateforme. Il oriente en outre le business model de l'écosystème, la plateforme étant l'élément structurant de ce dernier (Moore, 1996 ; Iansiti et Levien, 2004 ; Edouard et Gratacap, 2011 ; Isckia, 2011 ; De Vogeleer et Lescop, 2011). Dans les trois autres phases (expansion, autorité et régénération ${ }^{4}$ ) de l'écosystème, l'enjeu stratégique vise alors la stabilisation du business model élaboré en première phase. Cette stabilité repose d'une part sur la capacité des acteurs membres à créer une dépendance

\footnotetext{
${ }^{3}$ Les externalités de réseaux se manifestent lorsque le bénéficie qu'un agent retire de la consommation d'un bien ou service augmente avec le nombre des agents consommant le même type de bien (Katz et Shapiro, 1985).

${ }^{4}$ Selon le modèle de Moore (1996, p.103-109), à la phase expansion du cycle de vie de leur écosystème, les acteurs mettent en relation leurs composantes pour former un tout cohérent dont ils cherchent à en accroître la valeur et la performance. Tout au long de cette phase, l'enjeu stratégique des acteurs est de stabiliser le business model de l'écosystème qui laisse place en phase autorité à un jeu de coévolution autour du rôle de leader.
} 
mutuelle, et d'autre part sur la capacité du leader à investir en continu dans le développement et l'amélioration de sa plateforme (Moore, 1996).

\subsection{Les écosystèmes 'plateformes', un business model partiellement ouvert}

Pour encourager la connexion d'innovations externes et complémentaires à son produit, les droits de propriété intellectuelle de certaines composantes de la plateforme sont partagés avec les acteurs externes. La plateforme peut adopter un business model 'partiellement ouvert', en fonction de la liberté d'utilisation, de développement et de commercialisation octroyée par son propriétaire (West, 2003). Selon les possibilités octroyées, le degré de liberté et les obligations précisées dans le cadre de contrats ou de règles d'utilisation, le business model ouvert ou partiellement ouvert (BMO) de la plateforme peut être de type 'intermédiaire', 'produit' ou 'biface' (Boudreau et Lakahami, 2009; Eisenmann et al. 2011). L'identification de «qui vend à qui ? » d'une part, le positionnement et rôle endossé par la plateforme d'autre part, permettent de déterminer quel BMO est le plus adapté à cette dernière (Boudreau et Lakahami, 2009, p.72). Cette analyse permet en outre de préciser le degré d'autonomie accordé aux acteurs externes et d'identifier l'acteur qui contrôle la trajectoire d'innovation, décide du modèle de revenus et est directement en relation avec le consommateur final (cf. figure 1).

Figure 1. Plateforme technologique : typologie des business model ouverts

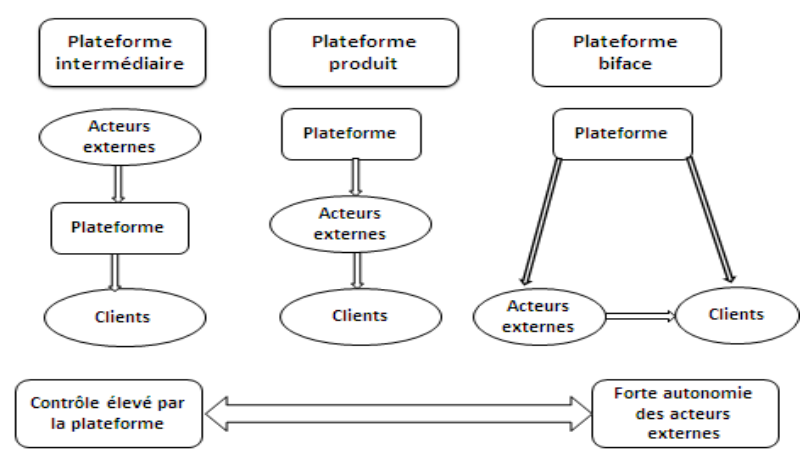

Source : Boudreau et Lakhani, 2009, p.73.

Lorsque les relations marchandes sont entretenues entre le propriétaire de la plateforme (vendeur) et les consommateurs (acheteurs), le BMO est de type 'intermédiaire'. Dans ce cas, la plateforme exerce un niveau de contrôle élevé. Elle donne au propriétaire la possibilité d'imposer certaines spécifications techniques ou applications finales. Lorsqu'en revanche le BMO est de type 'produit', le pouvoir de contrôle exercé par la plateforme est plus faible. La plateforme est dans ce cas un support aux modules, services ou produits déployés par des acteurs externes qui en conservent les droits résiduels, fixent les prix et les vendent directement aux consommateurs. Ce degré d'autonomie est plus important dans les BMO 'bifaces' ${ }^{5}$ où aucune interaction directe entre le détenteur de la plateforme et les acteurs externes n'est nécessaire pendant le processus de conception, de développement et d'industrialisation d'un nouveau produit. Le propriétaire peut cependant fixer des règles d'affiliation à sa plateforme (Boudreau et Hagiu, 2009). La plateforme 'biface', en raison des externalités de réseaux qu'elle génère, permet à deux ou plusieurs groupes d'agents d'interagir (Rochet et Tirole, 2006). Le nombre de consommateurs (groupe 1) affiliés à la plateforme est déterminant pour les acteurs externes (groupe 2). L’enjeu pour le détenteur de

\footnotetext{
${ }^{5}$ Baptisés « Marchés à deux versants ou multifaces» (two-sided or multisided) par Rochet et Tirole (2006).
} 
la plateforme est alors de concevoir un modèle de revenu basé sur les effets positifs des externalités de réseaux, les acteurs du premier groupe étant généralement caractérisés par une plus faible propension à payer que les acteurs du deuxième. En définitif, les plateformes technologiques incarnent l'écosystème au sens où elles en structurent les mécanismes de création et de partage de valeur, i.e. son business model (Gawer et Cusumano, 2002, 2008 ; Evans et al. 2006 ; Iansiti et Levien, 2004a, 2006 ; Iyer et Davenport, 2008 ; Baldwin et Woodard, 2009 ; Isckia, 2011).

\section{Une recherche-intervention menée au sein de NFCampus}

Cette recherche pose la question de savoir comment identifier l'acteur leader d'un écosystème naissant. Pour répondre à cette interrogation, l'article s'appuie sur une étude de cas, méthode adaptée "lorsque se pose une question du type 'comment' ou 'pourquoi' à propos d'un ensemble contemporain d'événements sur lesquels le chercheur a peu ou pas de contrôle » (David, 2004 ; Yin, 1993, p.20). Le cas étudié, Nice Futur Campus (NFCampus), permet d'analyser un processus de changement organisationnel complexe (2.1) qui évolue dans le temps et qui implique les acteurs affectés par ce changement dans notre démarche de recherche constructiviste (2.2).

\subsection{Présentation du cas NFCampus}

Les écosystèmes du NFC regroupent des acteurs du segment traditionnel, des acteurs du segment des nouveaux entrants (en particulier des fournisseurs de services) et des nouveaux métiers tel que les gestionnaires des services NFC plus communément appelés : les Trusted Secure Manager (TSM) (Basole, 2009 ; Pastorelly et al. 2011). Au sein de cet écosystème, les fournisseurs de services sont hétérogènes et de taille différente. Parmi les services NFC proposés, certains s'adressent à la majorité de la population, d'autres concernent un plus petit nombre d'utilisateurs. Les services NFC déployés sont généralement localisés et peuvent avoir le territoire pour lieu d'exercice (Loilier et Malherbe, 2013, p.200-201 ; Attour et Rallet, 2014). Représentatif des écosystèmes NFC, et plus particulièrement du 'core business' des écosystèmes du NFC au sens de Loilier et Malherbe (2012 ; 2013, p.200), Nice Futur Campus (NFCampus) réunit l'Université de Nice Sophia-Antipolis (UNS), Extelia (filiale du groupe La Poste), Moneo, Veolia Transport, Orange Labs, Twinlix, Mobille Distillery et Cassis International autour de la conception d'une carte étudiante multiservice et multimodale. Au sein de cet écosystème, les acteurs ont été confrontés à un double enjeu. Le premier enjeu vise à concevoir une offre de service couplant des services de la 'vie étudiante" ${ }^{\text {, }}$ et des services de la 'vie quotidienne' composés :

- d'un abonnement à l'offre Cityzi ${ }^{7}$ commercialisé par Veolia-Transport ${ }^{8}$ dans la ville de Nice depuis 2010 sous deux formules :

○ un abonnement 'Cityzi pour vélo-bleu' (location de vélo public)

○ un abonnement 'Cityzi pour Tramway et bus',

- un service de micro-paiement Moneo utilisable aux restaurants universitaires, cafétérias des différents campus de l'UNS et auprès des commerçants de proximité niçois partenaires de NFCampus,

\footnotetext{
${ }^{6}$ Services de gestion de la vie scolaire (information Campus, bibliothèque, emploi du temps), accès aux locaux universitaires, recherche de stage et embauches, etc.

${ }^{7}$ Nice est la première ville française à proposer un service de transport sur mobile NFC. L'offre Cityzi engloble un service de titre de transport de Veolia qui donne la possibilité à tout utilisateur détenteur d'un mobile NFC Cityzi d'acheter son titre de transport via son téléphone mobile « où et quand il veut ». http://www.cityzi.fr/

${ }^{8}$ Par définition, les acteurs membres d'un écosystème peuvent également être membre d'un autre écosystème (Moore, 1996 ; Gueguen et Pellegrin-Boucher, 2005), comme c'est le cas ici pour Veolia-Transport.
} 
- un service Woomji de réception et utilisation de coupons de réductions commerciaux (ticketing-couponning) proposé par Extelia ;

Le deuxième objectif vise à développer et tester une solution alternative à la puce Subscriber Identity Module (SIM) qui vient en support aux applications NFC et qui permet de distribuer l'offre finale à l'utilisateur. Les services de NFCampus sont en effet distribués aux étudiants via :

- un téléphone Samsung compatible NFC équipé d'une carte SIM NFC Cityzi délivré par Orange et déjà commercialisé via les services NFC déployés par la ville de Nice,

- des supports alternatifs à la carte SIM NFC permettant de rendre un terminal mobile compatible NFC. Il s'agit des stickers Bluetooth ${ }^{9}$ (un autocollant) de Mobile Distillery sur lesquels sont adossés un add-on $\mathrm{NFC}^{10}$ fourni par Twinlix.

Caractérisée par un double enjeu d'innovation, une problématique importante était au cœur de la réflexion stratégique des acteurs de NFCampus : quel est le business model de l'offre ? Cette interrogation était d'autant plus cruciale qu'à la naissance de NFCampus, l'élément structurant l'écosystème-plateforme n'était pas préalablement identifié. Pour les acteurs, l'élément le plus à même d'endosser ce rôle sont les supports SIM et/ou Stickers Bluetooth. La question alors posée par les praticiens portait sur la tarification (le modèle de revenus) de l'emplacement loué par les fournisseurs de services auprès du fournisseur du support choisi par les étudiants-utilisateurs de l'offre. Partant de là, parallèlement et tout au long du développement et des expérimentations technologiques (Chesbrough et Rosenbloom, 2002), nous avons été impliquées au sein de NFCampus pour mener une réflexion stratégique, avec la participation active des acteurs, portant sur le business model de l'écosystème comme le détaille le paragraphe qui suit.

\subsection{Méthodologie}

Ancrée dans une démarche constructiviste (Chanal et al. 1997 ; David, 1999, David et al. 2000 ; Gavard-Perret et al. 2012, p.34), notre méthode de recherche s'est d'abord attachée à co-construire le problème de recherche avec les praticiens pour ensuite articuler les connaissances procédurales dans un modèle qui favorise la compréhension de processus complexes tels que l'émergence des écosystèmes NFC. Porteur du livrable «modèle économique » au sein du projet NFCampus, une compréhension des représentations des acteurs a d'abord été nécessaire pour construire l'objet de l'étude : le business model d'un écosystème naissant. Très proche de la recherche-intervention au sens où le chercheur élabore un modèle théorique (par la confrontation de ses connaissances théoriques avec le problème du terrain) qu'il met en œuvre et évalue sur le terrain (David, 2000 ; Savall, Zardet, 2005 ; Cappelletti, 2009), notre méthodologie relève d'une démarche de recherche ingénierique au sens où elle s'intéresse à un processus organisationnel complexe, multicritère, multi-acteur et multi-rationnel (Chanal et al. 1997). De plus, empreinte d'une volonté pédagogique pour faciliter l'appropriation des connaissances théoriques par les managers, notre démarche a eu pour premier objectif d'aider les acteurs à construire et définir les problèmes liés à l'élaboration du business model de NFCampus premièrement, puis à proposer un outil (une grille d'interprétation ici) «pour améliorer l'appropriation par les praticiens des connaissances (....) procédurales qui viennent se confronter avec les connaissances théoriques existantes et les enrichir » (ibid, p.3).

De nature constructiviste, notre posture nous a amené à privilégier une démarche de recueil de

\footnotetext{
${ }^{9}$ Autocollant (à coller au dos du téléphone mobile) qui comprend un module NFC capable de communiquer de manière sans fil (via Bluetooth) avec le téléphone mobile via une technologie 'applet Java' installée sur ce dernier.

${ }^{10}$ Un add-on NFC est un type de patch qui optimise le fonctionnent d'un élément en ajoutant une ou plusieurs fonctionnalités.
} 
données par l'observation participante active, méthode appropriée lorsque le statut d'observateur du chercheur est connu de tous au sein de l'organisation (David, 1999; Chanlat, 2005). Dans cette perspective nous nous sommes immergées dans le contexte de l'observation pour en développer une intelligence via l'appropriation du langage des acteurs avec lesquels nous étions en interaction régulière (Lukka, 2005). Plusieurs allers-retours entre la théorie et le terrain ont alors été nécessaires pour laisser de la place à l'interprétation, la validation et la reformulation d'hypothèses par des interviews répétées et la confrontation des réponses données. Cette posture constructiviste a été suivie dans les quatre volets de la recherche : recherches théoriques, entretiens avec les experts, enquêtes auprès des étudiantstesteurs et étude du cas NFCampus. Les deux axes théoriques de cette recherche (stratégies de plateforme et business model ouverts) ont été construits lentement, à mesure de l'avancée dans la conduite et l'analyse des entretiens avec les experts et du cas étudié. La collecte d'informations s'appuie sur des données secondaires issues de l'analyse des rapports d'activités du projet, de la collecte d'informations disponibles en ligne, des documents techniques mis à notre disposition par les praticiens, etc. Les données primaires ont été collectées en plusieurs temps et méthodes. Elles résultent de 15 entretiens semi-directifs d'une durée de $1 \mathrm{~h} 30$ avec les praticiens, de deux enquêtes menées auprès d'un échantillon de 53 étudiants-testeurs de l'offre NFCampus (interrogés à l'aide d'un questionnaire fermé administré par mail), des réunions auxquelles nous avons participé ( 24 réunions) ou que nous avons nous-même animé ( 3 sessions de travail sur les business models).

Plus précisément, les entretiens semi-directifs ont permis de pointer les thématiques considérées importantes par les acteurs de NFCampus. L'analyse du contenu thématique de ces informations (Bardin, 2003) nous ont conduit à identifier six thématiques ${ }^{11}$ consolidées en deux (stratégies de plateformes et business models ouverts) qui ont à leur tour guidé l'analyse des données primaires et secondaires. L'animation de groupes de travail sur le business model de l'offre NFCampus a mobilisée l'approche d'Osterwalder et Pigneur (2005), puis a été guidée par les méthodes d'élaboration des business model sur le terrain introduites par les récents travaux de Chanal et al. (2011). Ces méthodes relèvent du "prototypage stratégique » qui passe par la description de la proposition de valeur et l'analyse de l'architecture de valeur. La description de la proposition de valeur permet de souligner les sources de valeur ${ }^{12}$ pour le client. Analysée sur la base de six questionnements: «qui innove, qui produit, qui offre, qui a besoin, qui utilise, qui paie » (Roehrich et Llerena, 2011, p.201), l'architecture de valeur de l'écosystème permet ensuite d'identifier «qui vend à qui » (Boudreau et Lakhani, 2009, p.72). Comme le montre la partie 3 suivante, l'analyse des sources de création et de partage de valeur de NFCampus permet d'identifier quelle plateforme élève son propriétaire au rôle de leader d'une part, de caractériser le BMO de NFCampus d'autre part.

\section{NFCampus : identification du ou des acteurs leaders et caractérisation du BMO de l'écosystème}

\subsection{Proposition de valeur de NFCampus : description de l'offre et identification de la plateforme}

Au sein de NFCampus, l'UNS déploie les services 'vie étudiante' sur une architecture NFC résultante de la combinaison de deux versions antérieurement expérimentées dans le cadre des

\footnotetext{
${ }^{11}$ Ecosystèmes d'affaires, business model, open innovation, stratégies de plateforme, marchés biface, prototypage stratégique.

${ }^{12} \mathrm{La}$ notion de valeur peut faire ici référence à deux types de valeur (Roehrich et Llerena, 2011) : la valeur d'échange (disposition à payer du client) et la valeur d'usage (les bienfaits que le client espère tirer de l'usage du produit).
} 
projets NFC Container ${ }^{13}$ et Campus Nova ${ }^{14}$. En vue de générer des sources de valeur pour son segment cible (les étudiants), il est possible de compléter l'offre initiale par la connexion de services dits de la 'vie quotidienne' sur l'architecture NFC de l'UNS. Le déploiement de ce deuxième groupe de services implique trois acteurs: Extelia, Moneo, Veolia Transport. Techniquement, pour chaque service de la 'vie quotidienne' une application mobile NFC a été déployée et connectée à l'architecture NFC de l'UNS. Plus précisément, l'application NFC du service Woomji d'Extelia a été déployée par l'UNS puis mise à disposition d'Extelia dans le cadre de l'expérimentation NFCampus. De même, Extelia et l'UNS ont conjointement déployé l'application mobile, en connexion avec les services Woomji, sur laquelle le service Moneo a été logé. Les applications Woomji et Moneo ont pour fonction particulière la mise en relation des étudiants avec un deuxième groupe d'utilisateur final : les commerçants de proximité niçois, partenaires du projet NFCampus. L'emboîtement et l'interopérabilité avec les applications Cityzi de Veolia-Transport et Moneo est en outre essentiel à l'application Woomji (pour émettre par exemple des offres de réduction sur les tickets de transport lors de la consommation d'autres services auprès des commerçants de proximité). Une dépendance mutuelle caractérise donc les services de la 'vie quotidienne' (Moore, 1996).

Stratégiquement, l'architecture NFC des services 'vie étudiante' de l'UNS ouvre, aux acteurs des services de la 'vie quotidienne', l'accès à un nouveau marché (les étudiants) (Isckia, 2011 ; De Vogeleer, Lescop, 2011). Le développement d'un service micro-paiement mobile NFC au sein de l'offre NFCampus permet en effet à Moneo d'explorer un nouveau marché ${ }^{15}$ en vue de pallier au faible taux de pénétration constaté sur le marché des cartes de paiement 'plastiques'. Pour Extelia, il s'agit de déployer sur le marché du commerce mobile une offre de ticketing-couponning déjà existante sur le marché du commerce électronique. Pour VeoliaTransport, NFCampus permet de confirmer une position dominante sur le marché local niçois des transports en commun. Ainsi, les services 'vie quotidienne' enrichisse la valeur de l'offre de services 'vie étudiante' de l'UNS par la combinaison originale de propositions de valeur déjà existantes, initialement adoptées et utilisées par les étudiants sous la forme de cartes plastiques indépendantes (une carte étudiante, une carte Moneo ${ }^{16}$ et une carte de transports).

Au sein de ces services externes, les applications de Moneo et de Veolia Transport semblent être les services qui contribuent le plus à enrichir la proposition de valeur initiale de l'UNS (dématérialiser l'offre de services 'vie étudiante') et à en renforcer la crédibilité. Comme le soulignent les résultats de l'enquête menée à l'issue de la première expérimentation en septembre 2011 auprès des 53 étudiants-testeurs de l'offre NFCampus (cf. figure 2), les

\footnotetext{
${ }^{13}$ NFContainer est une architecture technique mettant à disposition des fournisseurs de services et des prestataires techniques un ensemble d'outils leur permettant de développer des applications (NFC) à destination des mobiles sans contact. Ces applications mobiles sont le support du service déployé par le fournisseur de services. Par exemple, chaque service de la 'vie étudiante' et de la 'vie quotidienne' de NFCampus est déployé sur une application NFC.

${ }^{14}$ L'architecture technique Campus Nova, développée par l'UNS en partenariat avec le Crédit Agricole, est une architecture de service de paiement par terminal mobile. Projet développé avec le Crédit Agricole : c'est un concept original de Porte-Monnaie Electronique (PME) sur téléphone mobile contenant, notamment, deux fonctionnalités innovatrices : l'échange de monnaie de Personne à Personne entre les 2 mobiles (le P2P) / le paiement de Personne à Commerçant dans les mêmes conditions (le P2M). Le système permet à des commerçants de donner des coupons de réductions à ses clients à partir de leur téléphone NFC.

${ }^{15}$ Il s'agit par ailleurs d'un service également initialement commercialisé sous la forme de carte plastique au sein même de l'UNS (mais sans la technologie NFC) et développé dans le cadre d'une offre de service public locale : la carte 'Bordeaux ma ville'.

http://www.bordeaux.fr/pgPresStand8.psml? nfpb=true\&_pageLabel=pgPresStand8\&classofcontent=presentatio $\underline{\text { nStandard\&id }=57733}$

${ }^{16}$ Il convient toutefois de préciser ici que la carte plastique étudiante de l'Université de Nice incorpore déjà la fonction de micro-paiement du Restaurant Universitaire du Centre Régional des Euvres Universitaires et Scolaires (CROUS). Cette offre résulte d'un partenariat entre le CROUS, Moneo et l'UNS.
} 
services Moneo et Cityzi pour Bus-Tramway sont véritablement les deux applications créatrices de valeur pour les utilisateurs. La valeur ${ }^{17}$ créée par les services de la 'vie quotidienne', en particulier les services de micro-paiement de Moneo et de transport de Veolia-Transport, est largement plus importante que celle générée par les services de la 'vie étudiante'.

Figure 2. Extrait des résultats de l'enquête Usage et déterminants d'adoption de NFCampus (2011)

Perte d'utilité de NFCampus
si le service suivant est supprimé

En conclusion, au sein de NFCampus l'architecture NFC des services de la 'vie étudiante' de l'UNS jouent donc le rôle d'assembleur des composantes de NFCampus, conformément aux trois prérogatives essentielles relatives à toute plateforme technologique structurant un écosystème (Gawer, 2009 ; Gawer et Cusumano 2002, 2008, 2010, 2012). Les services 'vie étudiante' permettent aux fournisseurs de services 'vie quotidienne' de déployer leurs actifs sur la plateforme de l'UNS. Comme le prévoit les termes du contrat de partenariat, les acteurs externes conservent les droits de propriété intellectuelle de leurs actifs et peuvent librement définir le modèle de revenus de leurs services qu'il facture directement aux étudiants. L'architecture NFC des services 'vie étudiante' de l'UNS joue donc bien le rôle de plateforme 'produit' au sens de Boudreau et Lakahni (2009).

\subsection{Une intelligence collective ouverte initiée par le fournisseur de la plateforme-produit de NFCampus}

Pour répondre à l'appel à projet IPER-SMSC (Innovation dans les Processus d'Entreprises par la RFID - Services Mobiles Sans Contact) lancé en mars 2009 par le Ministère de l'économie, de l'industrie et de l'emploi, l'UNS a défini la trajectoire d'innovation suivante : concevoir une carte étudiante à la fois multiservice (couplant services de la 'vie étudiante' et services de la 'vie quoitidenne') et multimodale (multiplicité des modes d'accès : téléphone mobile compatible NFC ou stickers-Bluetooth permettant de rendre un téléphone mobile compatible NFC). En plus de mettre en relation des fournisseurs de services externes, l'architecture NFC de l'UNS suit un deuxième objectif d'innovation : expérimenter un support sécurisé alternatif à la carte SIM-Centric des téléphones mobiles NFC. La conception et la distribution de l'offre NFCampus jusqu'à l'utilisateur final (les étudiants) résulte donc bien, au sens de Edouard, Gratacap (2011) et Isckia (2011), d'une innovation collective ouverte instaurée par l'UNS entre :

- les fournisseurs de services externes d'une part,

- les fournisseurs de services externes (Extelia, Moneo, Veolia-Transport), l'UNS et le TSM de NFCampus (Cassis International, gestionnaire des services et des applications NFC) d'autre part,

- le TSM et les fournisseurs des supports logeant les applications NFC des services 'vie étudiante' et 'vie quotidienne' (Orange Labs, fournisseur de la Carte Sim-Centric et

\footnotetext{
${ }^{17}$ La valeur usage au sens de Roehrich et Llerena (2011).
} 
Mobille Distillery, fournisseur des Stickers Bluetooth logeant le SE 'add-on NFC' de Twinlinx) enfin.

Concrètement, en plus des fournisseurs de services 'vie étudiante' et 'vie quotidienne', la diffusion de l'offre NFCampus est dépendante de trois autres acteurs : les fournisseurs du support sécurisé SIM NFC (Orange), des supports NFC alternatifs de type Stickers Bluetouth Add-on (Mobile Distillery et Twinlinx), du gestionnaire des services et des applications NFC (le TSM Cassis International). L'usage des services 'vie étudiante' et 'vie quotidienne' déployés sur l'architecture NFC de l'UNS passe en effet par la mise en relation de leurs applications NFC avec le support sécurisé choisi par l'utilisateur (les étudiants). Cette connexion entre les fournisseurs d'applications (services) NFC et les fournisseurs du support sécurisé est assurée via une technologie de gestion 'over the air' $\left(\mathrm{OTA}^{18}\right)$ du TSM Cassis International comme le montre la figure 3 suivante.

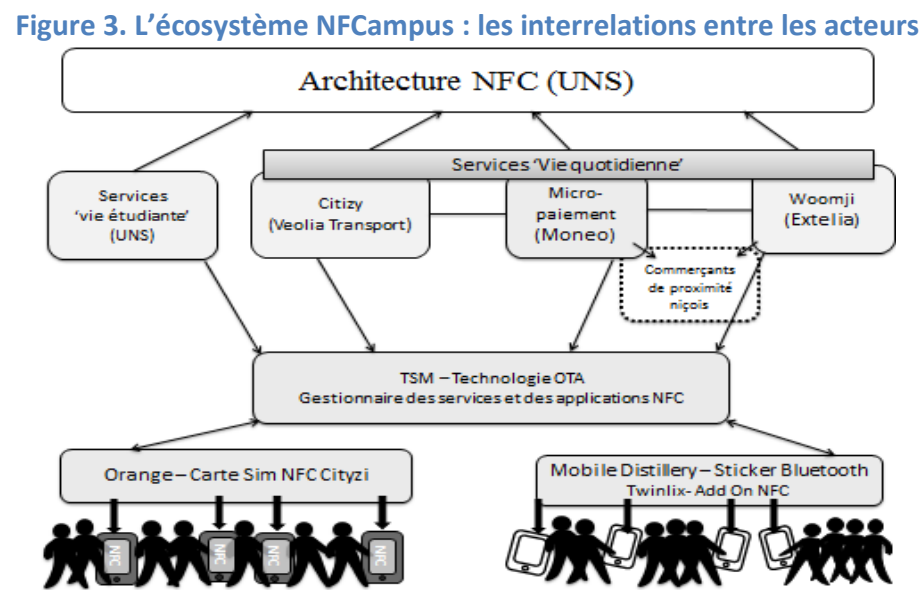

La technologie OTA permet au gestionnaire des services NFC de sécuriser les échanges de données entre fournisseurs de services NFC et leur fournisseur du support mobile sécurisé. Techniquement, il installe les applications NFC dans le support mobile sécurisé choisi par l'utilisateur final et gère leur cycle de vie. Cette activité permet au TSM d'enrichir considérablement la proposition de valeur de l'offre NFCampus qui peut alors être diffusée auprès des étudiants via différents canaux de distribution : la carte SIM-NFC Cityzi ou un support alternatif de type Stickers ou add-on NFC. Le TSM occupe donc bien, au sens de Basole (2009), un rôle d'intermédiaire (broker) entre les fournisseurs de services NFC et les fournisseurs du support mobile sécurisé qui assurent la connectivité de l'écosystème (bridge).

«On fait l'intermédiaire (le pont) entre les fournisseurs de services NFC (UNS, MONEO, Extelia, etc.) et les opérateurs de télécommunication. On assure une connexion entre différentes passerelles. L'enjeu est de fournir l'accès 'opérateur de télécommunication' aux prestataires de services et d'en assurer la maintenance. Par exemple, si le support des applications NFC est la carte SIM-Centric d'un opérateur mobile, les fournisseurs de services peuvent être bloqués car ils auront besoin d'un accord commercial avec l'opérateur de télécommunication. Or cette mise en relation entre fournisseurs de services et opérateurs de télécommunications est assurée nativement avec la plateforme OTA du TSM. ». Jean-Luc Garnier, Cassis International.

Au sein des écosystèmes-plateformes de type NFC, le leadership semble donc davantage être exercé par le segment des nouveaux entrants que par les fondateurs technologiques

\footnotetext{
18 «L'OTA (pour Over The Air) est une technologie permettant d'accéder aux données d'une carte SIM à distance. Il permet par exemple ainsi à un opérateur de téléphonie mobile de mettre à jour le contenu ou d'introduire un nouveau service sur tout un lot de cartes SIM de manière rapide, efficace et peu coûteuse ». (Wikipedia, www.wikipedia.org).
} 
(opérateurs de télécommunication et fournisseurs du support mobile sécurisé). Parmi les acteurs du segment des nouveaux entrants, on distingue premièrement les fournisseurs de plateforme technologique de type OTA qui endossent le rôle d'intermédiaire clé (brokers) entre les fournisseurs de services NFC et les fondateurs technologiques - connecteurs (bridge) de l'écosystème (Basole, 2009). On distingue deuxièmement les fournisseurs de services NFC, lesquels sont caractérisés par un nombre important de liens à la fois au sein de leur groupe mais aussi avec les autres membres de l'écosystème. Détenteurs d'une architecture technologique ou de services endossant une fonction de plateforme, les fournisseurs de services NFC sont les acteurs candidats au rôle de leader. Comme le soulignent les paragraphes 3.1 et 3.2, la plateforme-produit de l'UNS assure une fonction pivot, mais essentiellement en phase naissance de NFCampus. Comme tend à le montrer l'analyse de l'architecture de valeur de NFCampus dans le paragraphe suivant, ce positionnement stratégique de l'UNS est naturellement cédé aux fournisseurs de services 'vie quotidienne' dès la deuxième phase du cycle de vie de l'écosystème.

\subsection{Leadership et coexistence de plateformes produit et biface au sein de NFCampus}

Comme le montre l'analyse de l'architecture de valeur de NFCampus (figure 4), l'UNS se positionne à la fois sur la chaîne de valeur et sur la chaîne de demande. Plus précisément, elle se positionne en tant qu'acteur «qui a besoin » d'enrichir la valeur de sa plateforme-produit auprès d'un groupe d'utilisateur cible (les étudiants).

Figure 4. Analyse de l'architecture de valeur de NFCampus

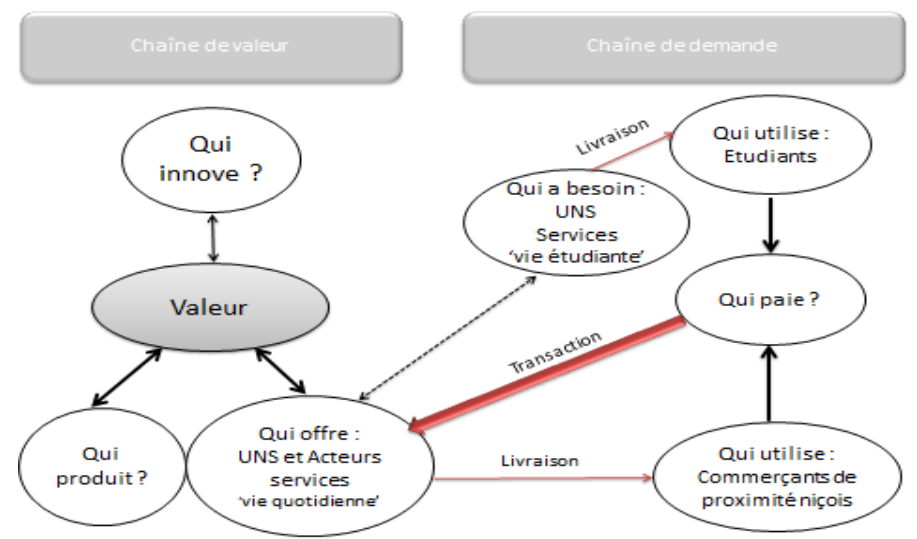

Adaptée de Roehrich et Llerena (2011, p.206) et appliqué au cas NFCampus

Pour satisfaire ce besoin, des acteurs externes déploient sur la plateforme-produit des services 'vie quotidienne' (Extelia, Moneo, Veolia-Transport) «qui offrent » des solutions (de micropaiement, de dématérialisation de leur carte d'abonnement-transport, etc.) accessibles aux clients cible de l'UNS (les étudiants) affiliés à la plateforme-produit de l'UNS. La valeur créée par ces services externes tient dans la mise en relation des étudiants avec un deuxième groupe d'utilisateurs des services 'vie quotidienne' (via les services Moneo et Woomji notamment) : les commerçants de proximité niçois. Pour ce deuxième groupe d'utilisateurs, le nombre d'étudiants affiliés à la plateforme-produit de l'UNS, et aux services externes de cette dernière, détermine leur adhésion aux bouquets de services 'vie quotidienne'. En effet, les services externes à la plateforme-produit de l'UNS offrent aux commerçants de proximité niçois la possibilité d'attirer un groupe de consommateurs de taille relativement intéressante (les étudiants) via des offres promotionnelles (service de ticketing-couponning Woomji d'Extelia) d'une part, un moyen de paiement supplémentaire (micro-paiement Moneo) d'autre part. En définitif, les services extérieurs à la plateforme-produit de l'UNS endossent le rôle de 
plateforme-biface entre deux groupes de clients «qui utilisent» l'offre NFCampus: les étudiants de l'UNS et les commerçants niçois (cf. figure 4). Plus le nombre d'étudiants adhérents à l'offre NFCampus sera élevé et plus le nombre de commerçants susceptibles d'accepter les terminaux de micro-paiement Moneo (comme mode de paiement supplémentaire aux moyens déjà utilisés par leur enseigne) et le service de ticketingcouponning Woomji ${ }^{19}$ peut augmenter.
«Via Woomji nous offrons aux commerçants niçois (restaurants et organisateurs d'événements en particulier) l'accès à une base de plusieurs milliers de clients».
Morgan Trevily, Extelia.
"Plus les étudiants seront nombreux à utiliser nos services via NFCampus, plus la base client que nous souhaitons vendre aux commerçants niçois sera attractive pour eux ». Jean-Luc Fretard, Extelia. "Pour convaincre les commerçants niçois d'utiliser nos terminaux de micro-paiement, il faut leurs assurés un bon potentiel d'utilisateurs».
Olivier Meric, Moneo.

En effet, le nombre d'étudiants utilisateurs de l'offre NFCampus sera d'autant plus déterminant à l'adhésion des commerçants que ce deuxième groupe d'utilisateur est identifié comme étant la face-payeur. Les étudiants ont en effet une faible disposition à payer l'accès à l'offre NFCampus : parmi les 53 étudiants-testeurs interrogés en septembre 2011, seulement $40 \%$ sont prêts à payer un accès au service de NFCampus ${ }^{20}$.

\begin{abstract}
«Nous allons adopter le même business model que celui adopté pour la carte étudiante (plastique) Moneo facturée aux banques ». Olivier Meric, Moneo.

«Comme le suggère les résultats de l'enquête et notre position sur la chaîne de valeur de NFCampus, nous réfléchirons à une tarification forfaitaire que nous appliquerons aux commerçants de proximité niçois, conscients que pour les étudiants de l'UNS le service doit être gratuit ».
\end{abstract}

Morgan Trevily, Extelia.

Les services Moneo et Woomji constituent donc bien des marchés bifaces au sens de Rochet, Tirole (2006) et Boudreau, Lakhani (2009). Etudiants comme commerçants ne sont en aucun cas impliqués dans le processus de conception, de développement ou d'industrialisation des services externes à la plateforme-produit de l'UNS. En revanche l'intensité d'utilisation de ces services externes est déterminante à la survie de l'offre finale. Il apparaît donc qu'en phases expansion et autorité de l'écosystème NFCampus, la fonction de plateforme biface endossée par un ou plusieurs services 'vie quotidienne' succède au rôle pivot exercé par la plateforme-produit de l'UNS tout au long de la phase naissance. En conclusion, le déploiement de l'offre NFCampus repose sur la mise en relation de deux types de plateformes au rôle pivot distinctement endossé en phase naissance par la plateforme-produit de l'UNS puis par une ou plusieurs plateformes bifaces en phases expansion et autorité.

\title{
Conclusion
}

La présente recherche s'est intéressée au cas des écosystèmes-plateformes naissants où, contrairement au cas traditionnellement étudiés par la littérature, le leader et la plateforme

\footnotetext{
${ }^{19}$ Comme le soulignent les résultats de l'enquête menée auprès des étudiants-testeurs de l'UNS, le service Moneo est clairement une plus grande source de création de valeur que l'offre Woomji (cf. figure 2 plus haut). Dans cet article, nous nous limitons à notre objet d'analyse : caractériser le rôle des acteurs de NFCampus pour comprendre comment en identifier l'acteur. L'adoption ou non de tous les services, et par conséquent la conception de l'offre de services finale pourra faire l'objet d'une recherche ultérieure. Il convient par ailleurs de souligner qu'au moment de l'enquête, les commerçants adhérents et proposant des réductions via le service Woomji étaient peu nombreux, restreignant probablement par-là l'utilisation du service par les étudiants.

${ }^{20}$ Plus précisément, parmi les étudiants-testeurs disposés à payer l'accès à l'offre NFCampus, $48 \%$ sont disposés à payer une contribution de 5 euros, $7 \%$ accepteraient de dépenser 10 euros, $5 \%$ consisteraient à payer 20 euros et seulement $2 \%$ envisageraient de payer plus de 20 euros.
} 
pivot de l'écosystème ne sont pas préalablement identifiés. Il pose la question de savoir comment identifier l'acteur leader d'un écosystème naissant. Dans cette perspective, il a appliqué à une innovation inter-organisationnelle les méthodes de prototypage stratégique jusque-là mobilisées au cas d'une seule firme. Analysée à l'aide d'une grille de lecture sur le BMO des plateformes technologiques, l'architecture de valeur de NFCampus permet de répondre aux travaux de Basole (2009) en montrant que, au sein des écosystèmes du secteur des télécommunications caractérisés par une offre de service territorialisée et destinée à une petite communauté d'utilisateurs tels que les écosystèmes du NFC (Loilier et Malherbe, 2013, p.201 ; Attour et Rallet, 2014), plusieurs acteurs pivots et leaders interagissent. Contrairement aux idées reçues des praticiens et conformément aux travaux de Basole (2009), cette guerre de leadership est observée entre les fournisseurs de plateformes. Cet article clarifie toutefois le positionnement des fournisseurs de plateformes. Les fournisseurs de plateformes candidats au rôle de leader occupent un rôle pivot $(h u b)$ et ne peuvent pas être, dans le cas des écosystèmes du NFC, les fournisseurs de plateformes intermédiaires (brokers).

Analysé dans les trois phases du cycle de vie de l'écosystème (Moore, 1996), le leadership des acteurs pivots diffère de la phase naissance aux phases expansion et autorité. Comme le montre le cas NFCampus, le premier acteur pivot émerge dès la naissance de l'écosystème (ouverture de la plateforme-produit de l'UNS). Ce positionnement stratégique est ensuite 'naturellement' cédé à un ou plusieurs acteurs externes à la plateforme-produit dans les phases expansions et autorité de l'écosystème. En effet, malgré la fonction pivot de sa plateformeproduit (l'architecture NFC de ses services 'vie étudiante') d'une part, ses nombreuses interactions avec les autres membres de l'écosystème d'autre part, l'UNS n'est que temporairement leader de l'écosystème. Elle assure un leadership tout au long de la naissance de NFCampus. En phase d'expansion, également positionnée sur la chaîne de demande, la nature biface des services complémentaires à sa plateforme-produit positionne alors les acteurs externes dans le rôle de leader. Parmi les acteurs leaders de la phase expansion, comme le laissent présager les résultats des enquêtes menées auprès des étudiants-testeurs, l'intensité d'utilisation des services bifaces déterminera quel service-pivot est crucial à l'offre NFCampus. L'enrichissement de la valeur de la plateforme-produit détenue par le leader identifié en phase naissance de l'écosystème tient en effet dans la nature même des actifs ou services complémentaires déployés par les acteurs externes, lesquels endossent le rôle de plateforme biface au sens de Boudreau et Lakhani (2009). En conclusion, et comme le montre les analyses de la proposition et de l'architecture de valeur de NFCampus, l'émergence d'un écosystème du NFC repose sur la coexistence de deux plateformes pivots caractérisées par des BMO : une plateforme-produit et une plateforme-biface. Ces plateformes octroient à leur(s) propriétaire(s) le rôle de leader, mais dans des phases distinctes du cycle de vie de l'écosystème.

Par ailleurs, contrairement au cas des écosystèmes-plateformes caractérisés par une seule plateforme pivot (Gawer et Cusumano, 2002 ; Iansiti et Levien, 2004; Gawer et Henderson, 2007; Eisenmann et al. 2009, 2011 ; De Vogeleer et Lescop, 2011 ; Isckia, 2011), le propriétaire de la plateforme conditionnant l'émergence d'un écosystème n'est pas nécessairement un acteur motivé par une stratégie de leadership ou de réaction à un échec de marché. Il peut être, comme c'est le cas pour l'UNS, motivé par des enjeux d'innovation organisationnelle ou d'innovation dans les outils de communication mis à disposition de ces utilisateurs. Par conséquent, sachant que les écosystèmes du NFC ont le territoire pour lieu d'exercice (Loilier et Malherbe, 2013 ; Attour et Rallet, 2014), le rôle des acteurs publics locaux est une condition essentielle à l'émergence et au déploiement d'une offre de services NFC. En d'autres termes, identifier et mettre à disposition des nouveaux entrants dans l'écosystème du NFC (opérateurs bancaires, de transports, etc.) un produit ou service assurant le rôle de plateforme-produit est une prérogative essentielle qu'un acteur public local doit 
assurer pour faciliter le déploiement d'une offre de services NFC sur son territoire. Ces résultats stimulants appellent néanmoins à élargir la présente, simple mais illustrative, étude de cas menée dans le cadre de cette recherche. Une analyse comparative analysant le cas d'écosystèmes numériques désormais existants tels que ceux déployés à Nice, Strasbourg (pour les offres Cityzi) ou Bordeaux (pour la carte bordeaux ma ville) permettrait de confirmer ou préciser quel acteur parmi les fournisseurs de services-bifaces tend à se positionner leader au sein de son écosystème NFC. Il conviendrait pour cela de s'intéresser à la manière dont l'interopérabilité de ces services-bifaces est rendue possible. Cette problématique semble en effet poser la question du management des droits de propriété intellectuelle relatifs aux actifs complémentaires composants une innovation interorganisationnelle dont la source de création de valeur tient dans l'interopérabilité des services qui la composent.

\section{Bibliographie}

ATTOUR A., RALLET A. (2014), « Le rôle des territoires dans le développement des systèmes trans-sectoriels d'innovation locaux : le cas des smart cities», Innovations $\mathrm{n}^{\circ} 43$, 2014/1, p.253-279.

BASOLE R.C., ROUSE W.B. (2008), «Complexity of service value networks: conceptualization and empirical investigation», IBM Systems Journal, Vol.47, No.1, p.5370.

BASOLE R.C. (2009), "Visualization of interfirm relations in a converging mobile ecosystem», Journal of Information Technology, No.00, p.1-16.

BARDIN L. (2003), L'analyse du contenu, PUF, Paris.

BALDWIN C., WOODARD C.J. (2009), «The architecture of platforms: a unified view», in Gawer A. (ed.), Platforms, Markets and Innovation, Edward Elgar, London, UK, p.19-44.

BOUDREAU K. (2010), «Open Platform Strategies and Innovation: Granting Access vs. Devolving Control», Management Science, vol. 56, n¹0, pp.1849-1872.

BOUDREAU K., HAGIU A. (2009), «Platform Rules: multi-sided platforms as regulators», in Gawer A. (ed.), Platforms, Markets and Innovation, Edward Elgar, London, UK, p.163191.

BOUDREAU K., LAKHANI K. (2009), «How to Manage Outside Innovation: Competitive Markets or Collaborative Communities?», MIT Sloan Management Review, Vol. 50, No.4, p.69-75.

CAPPELLETTI L. (2009), «Performing an internal control function to sustain SOX 404 and improve risk management: Evidence from Europe », Management Accounting Quaterly, Summer, Vol. 10, No.4, p.17-27.

CHANAL V., LESCA H., MARTINET A.C. (1997), «Vers une ingénierie de la recherche en sciences de gestion », Revue Française de Gestion, No.116, p.41-51, nov.-déc.

CHANAL V., GIANNELLONI J.L., PARENT R. (2011), «Construire un business model rentable basé sur la gratuité », in Chanal V. (éd.), Business models dans l'innovation, Pug, Presses universitaires de Grenoble, p.73-85.

CHANLAT J. (2005), «La recherche en gestion et les méthodes ethnosociologiques», in Roussel P., Wacheux F., Management des ressources humaines : méthodes de recherche en sciences humaines et sociales, De Boeck, Paris, p. 159-175.

CHESBROUGH H. (2006), Open Business Models: How to Thrive in the New Innovation Landscape, Boston: Harvard Business School Press

CHESBROUGH H., ROSENBLOOM R.S. (2002), «The role of the business model in capturing value from innovations : evidence from Xerox Corporation's technology 
DAVID A. (1999), «Logique, épistémologie et méthodologie en sciences de gestion», Conférence de l'AIMS.

DAVID A., Hatchuel A., Laufer R. (2000), Les nouvelles fondations des sciences de gestion. Vuibert, Paris, p.125-139.

DAVID A. (2004), «Etudes de cas et généralisation scientifique en sciences de gestion», Actes de la XIIIème Conférence de l'Association Internationale de Management Stratégique, Le Havre.

DE VOGELEER E., LESCOP D. (2011), «Plateformes, coordination et incitations», Management \& Avenir, No.46, 2011/6, p.200-218.

EDOUARD S., GRATACAP A. (2009), «De l'entreprise virtuelle à l'entreprise écosystèmique», Vie \&Sciences de l'entreprise, No.181, 2009/1, p.27-40.

EDOUARD S., GRATACAP A. (2011), "Proposition d'un modèle d'intelligence collective pour les écosystèmes d'affaires », Management \& Avenir, No.46, 2011/6, p.177-199.

EISENMANN T.R., PARKER G., VAN ALSTYNE M.W. (2009), «Opening platforms: how, when and why? », in Platforms, Markets and Innovation, A.Gawer (ed.), Northampton, MA: Edward Elgar, p.131-162.

EISENMANN T.R., PARKER G., VAN ALSTYNE M.W. (2011), «Platform envelopment», Strategic Management Journal, Vol.32, No.12, p.1270-1285.

EVANS D.S., HAGIU A., SCHMALENSEE R. (2006), «Invisible engines: how software platforms drive innovation and transform industries», First MIT Press paperback edition.

GAVARD-PERRET M.-L., GOTTELAND D., HAON C., JOLIBERT, A. (2012), Méthodologie de recherche. Réussir son mémoire ou sa thèse en sciences de gestion,Pearson Education France, Paris.

GAWER A. (2009), «Platforms dynamics and strategies: From products to services», in Gawer A. (ed.), Platforms, Markets and Innovation, p. 45-76.

GAWER A., CUSUMANO M.A. (2002), «Platform leadership : How Intel, Microsoft and Cisco drived industry innovation», Harvard Business School Press .

GAWER A., CUSUMANO M.A. (2008), «How firms become platform leaders », MIT Sloan Management Review, Vol.49, No.2, p.28-35.

GAWER A., HENDERSON. R. (2007) "Platform Owner Entry and Innovation in Complementary Markets: Evidence from Intel", Journal of Economics and Management Strategy, Wiley Blackwell, vol. 16, $\mathrm{n}^{\circ}$ 1, pp. 1-34.

GAWER A., CUSUMANO M.A. (2010), «The organization of technological platforms», Research in the Sociology of Organizations, Vol.29, p. 287-296.

GAWER A., CUSUMANO M.A. (2012), «Industry platforms and ecosystem innovation», DRUID 2012. CBS, Copenhagen, Denmark.

GUEGUEN G., PASSEBOIS-DUCROS J. (2011), "Les écosystèmes d'affaires : entre communauté et réseau», Management \& Avenir, No.46, 2011/6, p.131-156.

HAGIU A. (2007a), "Merchant or two-sided platform? », Review of Network Economics, Vol.6, No.2, p. 115-133.

HAGIU A. (2007b), "Multi sided platforms: From microfoundations to design and expansion strategies», Harvard Business School, Working Paper No. 07-094.

HAGIU A. (2009), «Proprietary vs.Open two-sided platforms and social efficiency», Harvard Business School Working Paper 09-113.

IANSITI M., LEVIEN R. (2004), The keystone advantage: what the new dynamics of business ecosystems mean for strategy, innovation, and sustainability, HBS Press, Boston.

ISCKIA T. (2009), "Amazon's evolving ecosystem: A cyber-bookstore and Application Service Provider», Canadian Journal of Administrative Sciences, Vol.26, No.4, p.332-343. 
ISCKIA T. (2011), « Ecosystème d'affaires, stratégies de plateforme et innovation ouverte : vers une approche intégrée de la dynamique d'innovation », Management \& Avenir, No.46, 2011/6, p.157-176.

ISCKIA T., LESCOP D. (2009), «Open innovation within business ecosystems: a tale from Amazon. com. », Communications and Strategies, ${ }^{\circ} 74$, p.37-54.

ISCKIA T., LESCOP D. (2013), «Platform-based Ecosystems: Leveraging Network Centric Innovation», in S. Ben Letaïfa, A. Gratacap and T. Isckia (eds), Understanding Business Ecosystems: How Firms Succeed in the New World of Convergence, De Boeck, p.97-111.

IYER B., DAVENPORT T. (2008), «Reverse engineering google's innovation machine», Havard Business Review, Vol.86, No.4, p.58-56.

IYER B., LEE C.H., VENKATRAMAN. (2006), "Managing in a "Small World Ecosystem": Lessons from the software sector», California Management Review, Vol.48, No.3, p.28-47.

KATZ M., SHAPIRO C. (1985), « Network Externalities, Competition and Compatibility», American Economic Review, Vol. 75, No.3, p. 424-440.

KOENING G. (2012), «Le concept d'écosystème d'affaires revisité», M@n@gement, Vol.15, (2012)/2, p.209-224.

KOEINING G. (2013), «Business ecosystem revisited», in Ben Letaifa S. (ed.), Understanding business ecosystems, De Boeck, Paris, p.69-84.

LOILIER T., MALHERBE M. (2012), «Le développement des compétences écosystèmiques. Le cas de l'ESA émergent des services mobiles sans contact», Revue Française de Gestion, No.222, 2012/3, p.89-105.

LOILIER T., MALHERBE M. (2013), «Experimentation and the development of ecosystemic competencies in the field of contactless mobile services», in Ben Letaifa S. (ed.), Understanding business ecosystems, De Boeck, Paris, p.193-207.

LUKKA K. (2005), " Approaches to case research in management accounting: the nature of empirical intervention and theory linkage», in Jönsson S., Mouristen J. (eds), Accounting in Scandinavia-The northern lights, Liber\&Copenhagen Business School Press, p.375-399.

MOORE J.F. (1996), The death of Competition - Leadership and Strategy in the age of business ecosystem, Harper Business, New York.

OSTERWALDER A., PIGNEUR Y. (2005), «Clarifying business models: origins, present and future of the concept», Communication of AIS, 2005 (16), pp.1-25.

PARKER G., VAN ALSTYNE M. (2005), «Two-sided network effects : A theory of information product design» Management Science, Vol.51, No.10., p.1494-1504.

PASTORELLY N., BENOUALI H ., LEBLANC C., MIRANDA S., ATTOUR A. (2011), «Nice Futur Campus, un bouquet de services NFC dans une carte virtuelle étudiant», RTSI- Ingénierie des systèmes d'information, Vol.16, №4/2011, p.63-86.

ROCHET J.C., TIROLE J. (2006), « Two-Sided Market: a progress report», The Rand Journal of Economics, Vol.3, No.35, p. 645-667.

ROEHRICH G., LLERENA D. (2011), «Du business model à l'émergence de nouveaux marchés », in Chanal (éd.), Business models dans l'innovation, Pug, Presses universitaires de Grenoble, p.185-209.

TEECE D.J. (2010), «Business models, business strategy and innovation », Long Range Planning, Vol.2-3, No.43, p.172-194.

WEST J. (2003), «How open is open enough? Melding proprietary and open source platform strategies», Research policy, Vol.32, No.7, p.1259-1285.

YIN R. (1993), Case study research: design and methods. Newbury Park (CA), Sage Publications. 